\title{
Ubiquitination-mediated protein degradation and modification: an emerging theme in plant-microbe interactions
}

\author{
Li-Rong Zeng ${ }^{1}$, Miguel E Vega-Sánchez ${ }^{1}$, Tong Zhu², Guo-Liang Wang ${ }^{1,3}$ \\ ${ }^{I}$ Department of Plant Pathology and Plant Molecular Biology and Biotechnology Program, The Ohio State University, Columbus, \\ OH 43210, USA; ${ }^{2}$ Syngenta Biotechnology Inc, Research Triangle Park, NC 27709-2257, USA; ${ }^{3}$ Rice Genomics Laboratory, Hunan \\ Agricultural University, Changsha, Hunan 410128, China
}

Post-translational modification is central to protein stability and to the modulation of protein activity. Various types of protein modification, such as phosphorylation, methylation, acetylation, myristoylation, glycosylation, and ubiquitination, have been reported. Among them, ubiquitination distinguishes itself from others in that most of the ubiquitinated proteins are targeted to the $26 \mathrm{~S}$ proteasome for degradation. The ubiquitin/26S proteasome system constitutes the major protein degradation pathway in the cell. In recent years, the importance of the ubiquitination machinery in the control of numerous eukaryotic cellular functions has been increasingly appreciated. Increasing number of E3 ubiquitin ligases and their substrates, including a variety of essential cellular regulators have been identified. Studies in the past several years have revealed that the ubiquitination system is important for a broad range of plant developmental processes and responses to abiotic and biotic stresses. This review discusses recent advances in the functional analysis of ubiquitination-associated proteins from plants and pathogens that play important roles in plant-microbe interactions.

Cell Research (2006) 16:413-426. doi:10.1038/sj.cr.7310053; published online 15 May 2006

Keywords: Ubiquitination, defense response, plant-microbe interactions, U-box protein, Spl11

\section{Introduction}

Plants are routinely exploited by a spectrum of parasites, such as viruses, bacteria, fungi, nematodes, and insects in their life cycles. Unlike animals, plants are sessile and lack a somatically adaptive immune system in responding to pathogen infections and insect attacks. Instead, plants have evolved other multifaceted mechanisms to protect themselves from various biotic and abiotic challenges [1]. Both pre-formed defense barriers and induced defense responses are employed by plants to counteract potential pathogen invasion [2]. Constitutive barriers, such as cuticular wax layers, pre-formed antimicrobial enzymes, and secondary metabolites, could function as the first fortification that pathogens must penetrate or bypass. Induced

Correspondence: Guo-Liang Wang ${ }^{1}$, Li-Rong Zeng ${ }^{2}$

${ }^{1}$ Tel: 614-292-9280; Fax: 614-292-4455;

E-mail: wang.620@osu.edu

${ }^{2}$ Tel: 614-292-8231; Fax: 614-292-4455;

E-mail: zeng.17@osu.edu defense reactions are mounted when the pathogen attacks are perceived by the hosts. Such pathogen recognition and subsequent induction of defense responses is critical to the protection of plants if preformed barriers are overcome by an evolutionarily active population of pathogens, which is frequently the case in nature. Based on whether the attacked plants are natural hosts of the pathogen or not, resistance to pathogens can be divided at 'species' and 'cultivar' levels, respectively [3]. The species-level resistance, also known as non-host resistance, refers to the fact that any given pathogen can only cause disease on a limited range of plant species. The cultivar-level resistance is also called race-specific resistance. During the process of co-evolution with the pathogens, plants have evolved resistance $(R)$ genes to specifically recognize cognate avirulence (Avr) genes from the pathogens [4]. Such race-specific plant-pathogen interactions conform to the gene-for-gene model, in which the presence of a plant $\mathrm{R}$ protein and its corresponding pathogen-encoded $A v r$ gene product leads to resistance, while lack of functional products of either gene results in disease [5]. Recognition of the Avr gene product 


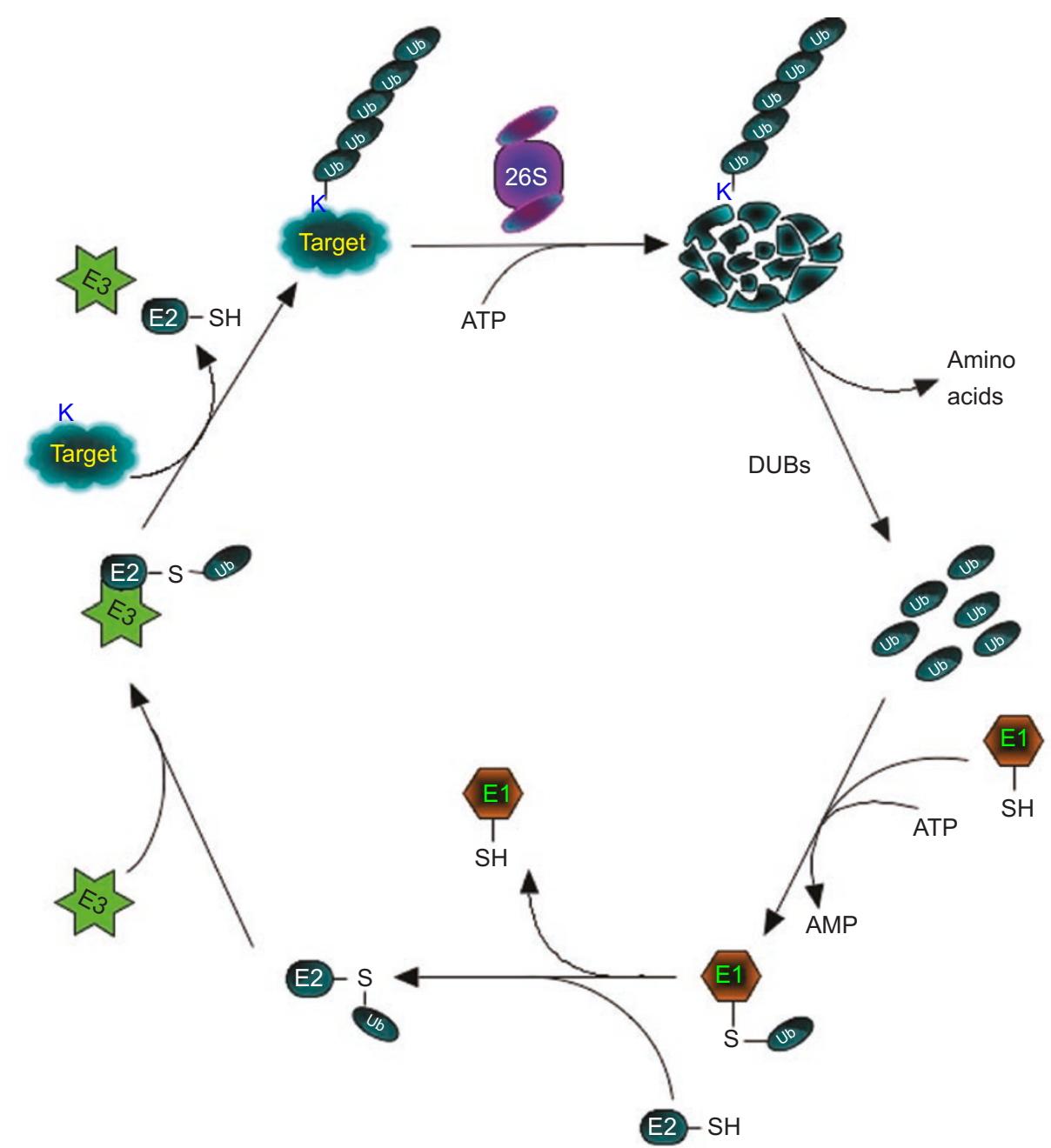

Figure 1 The Ubiquitin/26S proteasome pathway. The protein ubiquitination begins with the activation of the ubiquitin molecule $(\mathrm{Ub})$ in an ATP-dependent manner. The activated ubiquitin is then transferred to the active site of the ubiquitin-conjugating enzyme (E2). Finally, an ubiquitin-ligase (E3) binds E2 and catalyzes the formation of an isopeptide linkage between the activated ubiquitin and the Lysine residue of the substrate protein. After a chain of multiple ubiquitins is attached to the target protein, it is usually destined to the $26 \mathrm{~S}$ proteasome, where the target protein is degraded and the ubiquitin monomers are reclaimed by the action of de-ubiquitination enzymes (DUBs).

by the R protein triggers a complex of signal transduction cascade culminating at local (e.g. hypersensitive response (HR)) and global (e.g. systemic acquired resistance (SAR)) defense mobilization in the plant $[6,7]$. Over the years, the gene-for-gene model has been refined. In the "guard" hypothesis, R proteins only 'recognize' the Avr protein indirectly, for example after it complexes with other host protein(s), such as RIN4 in Arabidopsis [8, 9]. These host proteins are regarded as virulence targets. Through the binding of the pathogen Avr protein (also termed as effector) to the target proteins, a susceptible reaction ensues. In the case of resistance, it has been proposed that the $\mathrm{R}$ gene product evolved to recognize the Avr-dependent binding or modification of the host target protein (s) [10]. To date, over $50 R$ genes have been isolated from different plant species, and key components in the defense signal transduction pathways in $R$-gene-mediated resistance, such as enhanced disease susceptibility (EDS1) and required for MLA resistance1 $(R A R 1)$, have also been identified $[11,12]$. Extensive reviews of $R$ genes and $R$-gene-mediated defense signaling pathways are available elsewhere [13]. Here, we will focus our discussion on recent findings regarding the involvement of the ubiquitination machinery in plant-microbe interactions and plant defense signaling. 


\section{The ubiquitin/26S proteasome system}

Two distinct yet coordinate steps are involved in the degradation of a protein by the ubiquitin system: (i) covalently attaching multiple ubiquitin molecules to the target protein; (ii) degrading the modified protein by the $26 \mathrm{~S}$ proteasome (Figure 1). Ubiquitination of the target protein is achieved by three sequential enzymatic reactions that act in concert. Initially, the ubiquitin-activating enzyme, E1, catalyzes the activation of the ubiquitin molecule, an evolutionarily conserved protein of 76 residues, in its C-terminal Gly to a high-energy thiol ester intermediate. Following activation, an ubiquitin-conjugating enzyme, E2 (also called ubiquitin-carrier proteins, UBC), catalyzes the transfer of the activated ubiquitin moiety from E1 to a member of the ubiquitin-protein ligase (E3) family. E3 catalyzes the last step of the conjugation process, transferring the ubiquitin from the E2 to a lysine residue in the target protein, often by forming an intermediate complex with the E2 and the protein substrate. After the first ubiquitin moiety is transferred to the protein target, a polyubiquitin chain is then synthesized by transferring additional activated ubiquitin moieties to Lys48 of the previously conjugated ubiquitin molecule. The attached polyubiquitin chain most likely will serve as a signal recognized by the $26 \mathrm{~S}$ proteasome. In addition to the formation of the polyubiquitin chain through the Lys48, monomeric ubiquitylation and polyubiquitylation through the Lys 29 or Lys63 residues of ubiquitin have also been reported and it is thought that these alternative ubiquitination events may be involved in cellular processes other than degradation [14].

The 26S proteasome is a giant self-compartmentalized protease that is composed of a 20S barrel-shaped core particle (CP) with an inner proteolytic chamber and two flanking $19 \mathrm{~S}$ polar regulatory particles (RP), where protein recognition and ATP-dependent unfolding occur [15]. In eukaryotes, the 20S CP is composed of four stacked heptameric rings that are made of related $\alpha$ and $\beta$ subunits in an $\alpha_{1-7} / \beta_{1-7} / \beta_{1-7} / \alpha_{1-7}$ configuration [16]. The two central $\beta$-subunit heptameric rings form the catalytic chamber. Three of the $\beta$ subunits, $\beta 1, \beta 2$, and $\beta 5$, are post-translationally processed at their amino terminus to yield an active protease site. The $\alpha$-subunit rings at the two outer sides of the central $\beta$-subunit rings form a gated channel that controls the passage of the unfolded substrates and cleaved peptides in and out of the proteolytic chamber. The 19S RP particle is made of two subcomplexes, the lid and the base. The base subcomplex contacts with the a-subunit ring of the 20S CP and contains a ring of six AAA-ATPase subunits (RPT1-6) along with three non-ATPase subunits (RPN1, RPN2, and RPN10). Generally, the lid subcomplex encompasses eight stoichiometric subunits (RPN3, RPN5,
RPN6, RPN7, RPN8, RPN9, RPN11, and RPN12) [17]. However, some non-essential subunits may transiently bind to the lid subcomplex in regulating $26 \mathrm{~S}$ proteasome activity and localization $[18,19]$. In addition, the deubiquitinating enzymes (DUBs) that cleave the $\varepsilon$-amide bond between ubiquitin and a lysine side chain to recycle the ubiquitin molecules from the substrate proteins are also found in the 19S subcomplex [16]. Unlike the 20S CP, of which the structure, assembly, and enzymatic mechanism have been elucidated, the organization and specific role of most subunits of the 19S complex remain unknown [16]. Several substrate-recruiting proteins, probably including chaperones, have been implicated in helping transfer the ubiquitinated protein from the ubiquitination machinery to the $26 \mathrm{~S}$ proteasome [20].

\section{Diversity of plant E3 ligases}

One important feature of the ubiquitination pathway is its hierarchical structure in terms of the number of E1s, E2s, and E3s in a given genome. For example, the Arabidopsis genome encodes $2 \mathrm{E} 1 \mathrm{~s}, 41 \mathrm{E} 2 \mathrm{~s}$, and over $1200 \mathrm{E} 3$ components [21, 22]. In the ubiquitin system, E3 ubiquitin ligase plays a central role in selecting the myriad of appropriate candidate proteins during the ubiquitination process [23]. This is manifested by the fact that E3s are the most diverse components in the ubiquitination pathway [15]. Until recently, four different families of ubiquitin ligases had been described based on their subunit composition and mechanism of action: HECT, SCF, APC, and RING/U-box [22]. The HECT (homologous to $\underline{\mathrm{E}} 6-\mathrm{AP} \underline{\mathrm{COOH}}$ terminus) domain was initially identified in the viral E6-associated protein (E6-AP) during the study of human papilloma viruses $[24,25]$. It is composed of $\sim 350$ amino acids and its $\mathrm{C}$-terminus contains a conserved active cysteine residue required for the ligase function. The HECT-type ligase is the only known E3 that forms an E3-ubiquitin thiolester intermediate before the final attachment of ubiquitin to the substrate [24]. The SCF-type E3 ligase is typically composed of four different polypeptides: SKP1 that serves as an adaptor protein; Cullin1 (CDC53); RING-finger protein RBX1 (or ROC1 and HRT1) that interacts with the Cullin1 and E2-conjugating enzyme; and an F-box protein that is responsible for recruiting substrates. Structural analysis indicated that Cullin1 (most of the time abbreviated as CUL1) acts as a scaffold that directs other members of the SCF complex to bring the ubiquitin-loaded E2s and the substrate in proximity to promote the transferring of ubiquitin directly from the E2 to the substrates [26]. Besides Cullin1, recent data indicated that Cullin2 and Cullin3 also form similar groups of E3 ligases, in which an elongin C-SOCS module or a BTB protein replaces the SKP1-F-box protein module 
Table 1 Members of the ubiquitin/26S proteasome system implicated in plant defense against biotic and abiotic challenges

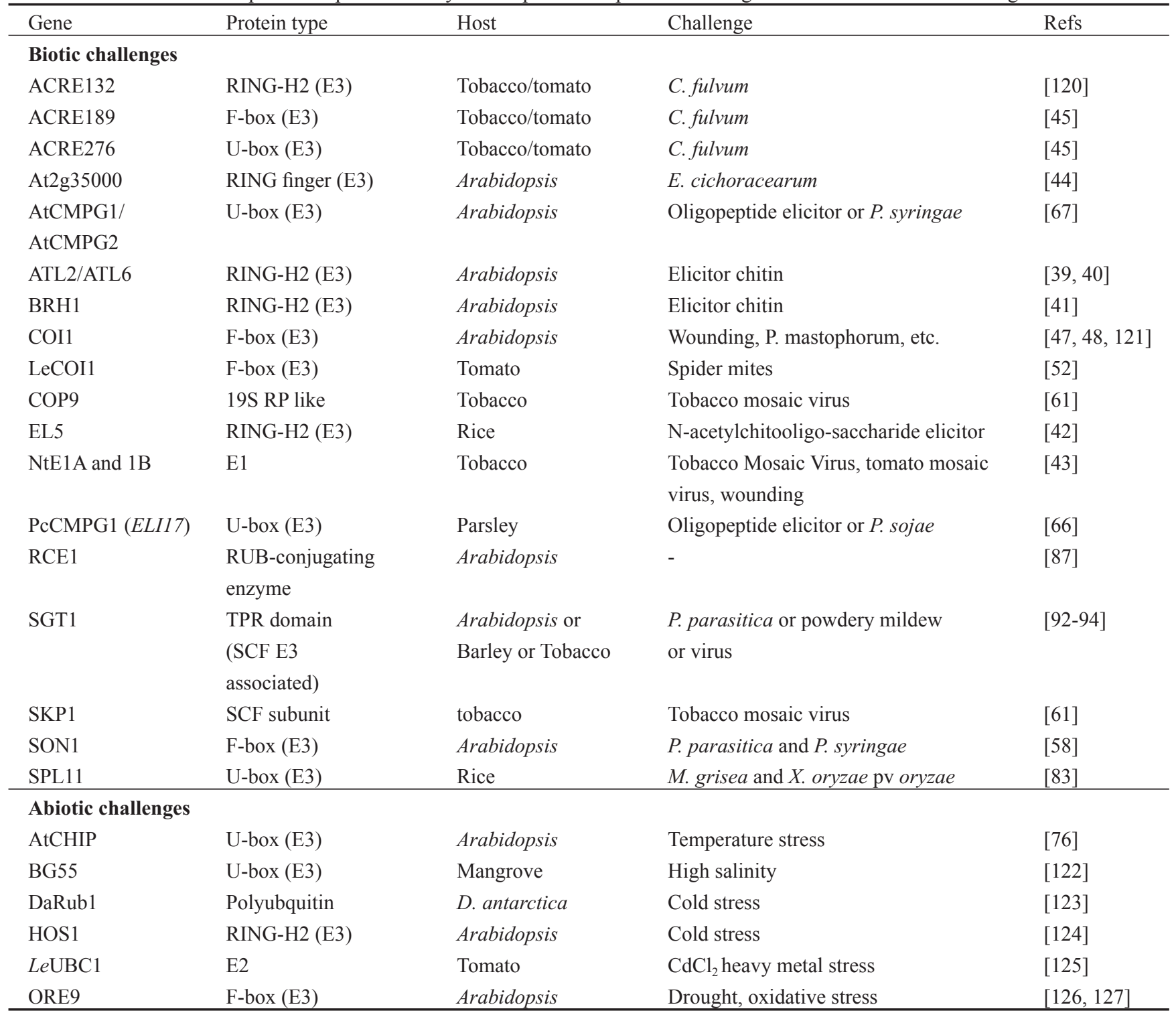

in the Cullin1-type SCF E3 ligase, respectively [27-29]. In the Arabidopsis genome, around 700 genes are predicted to encode proteins containing the 40 amino-acid F-box motif [30]. The anaphase promoting complex (APC)-type E3s are complexes of over 10 proteins. The complex also contains a Cullin protein, although their action mechanism is distinct from that of SCF group E3s. It is expected that only a single or a few APC isoforms exist in plants [22, 31]. The RING (really interesting new gene) finger motif is characterized by the presence of $\mathrm{Zn}^{2+}$-chelating amino acid residues hallmarked as $\mathrm{C} 3 \mathrm{HC} 4(\mathrm{C}$, cysteine; $\mathrm{H}$, histidine) or $\mathrm{C} 3 \mathrm{H} 2 \mathrm{C} 3$, which forms two cross-brace-arranged free loops. Like SCF E3s, the RING-type E3 ligases serve as scaffolds to bring together the activated ubiquitin-E2 and the substrate in promoting the transfer of ubiquitin to the substrate. In contrast to that only few HECT-type E3s are identified in plants [32], the SCF and the RING-type E3 are found in hundreds of proteins [33,34]. The U-box is a novel E3 ubiquitin ligase activity-related protein domain that was first identified in the yeast ubiquitination factor UFD2 [35]. The U-box contains $\sim 75$ amino acids and possesses a tertiary structure resembling that of the RING finger $[36,37]$. The major difference between the U-box and RING domains is that the U-box lacks the hallmark 
$\mathrm{Zn}^{2+}$-chelating cysteine and histidine residues characteristic of RING fingers. Consequently, the conserved zinc-binding residues supporting the cross-brace arrangement in RING-finger domains are replaced by hydrogen-bonding networks in the U-box [37].

\section{Members of the ubiquitination system are implicated in plant defense signaling}

By removing abnormal proteins, the ubiquitin/26S proteasome system performs an essential housekeeping role in the cell. Importantly, the system also serves as control/ regulation mechanism by eliminating normal proteins, such as rate-limiting enzymes and key regulators, in different pathways. The first clue of the involvement of ubiquitination in plant defense came from the observation that the Arabidopsis R protein RPM1 is degraded coincidently with the onset of the HR [38]. In the past several years, approximately a dozen ubiquitination-related components have been identified that are involved in plant-pathogen or plant-insect interactions (Table 1). These genes were identified from different plant species and most of them are induced after pathogen or elicitor challenges. For example, the RING-finger-type E3 ligases ATL2 and ATL6 were rapidly induced in Arabidopsis plants treated with chitin elicitor [39, 40]. Similarly, other members of RING-type E3 ligases such as EL5 from rice and BRH1 from Arabidopsis were also induced after elicitor treatment [41, 42]. The upregulation of these genes in plants suggested that they might be involved in the response to biotic stresses. However, whether they play a role in signaling or regulating plant defense remains to be determined. The induction of an E1 in tobacco plants after virus infection also supports the idea that ubiquitination is involved in plant-pathogen interactions [43]. Interestingly, the E1 enzymes NtE1A and $\mathrm{NtE} 1 \mathrm{~B}$ were induced in tobacco plants only when inoculated with tobacco mosaic virus (TMV) or tomato mosaic virus (ToMV), but not cucumber mosaic virus. In the examination of Arabidopsis global gene expression after treatment with chito-octamers and hydrolyzed chitin, Ramonell et al. [44] identified a RING-finger E3 ligase that showed early responsiveness to the elicitor treatment. Lossof-function of the gene by T-DNA insertional mutation resulted in enhanced disease resistance when Arabidopsis plants were inoculated with low-density of Erysiphe cichoracearum, demonstrating that this $\mathrm{E} 3$ ligase is required for plant responses to powdery mildew invasion, probably in the initial signaling process to pathogen infection.

As mentioned above, E3 ubiquitin ligases are the most abundant members of the ubiquitination system and they are generally considered to be most important in controlling substrate specificity. Therefore, it is not surprising that most of the components of the ubiquitination system that have been shown to play a role in the regulation of various pathways belong to the E3 group. Except for the HECT- and APC-type E3s, to which only a limited number of members have been identified in plants, all other classes of E3s have been implicated in plant defense. The ACRE132 (Avr9/ $\underline{\text { Cf }} 9$ rapidly elicited), $A C R E 189$, and $A C R E 276$ genes encode different types of E3 components. They were identified in a cDNA-AFLP analysis of Cf9 tobacco cell cultures treated with Avr9-containing intercellular fluid. Silencing of ACRE 189 and ACRE276 dramatically reduced Avr4/Cf4or $A v r 9 / C f 9$-induced HR, apparently suggesting that they are involved in the signaling of $C f 4$ - and $C f 9$-mediated cell death and/or resistance [45]. However, further studies, such as phenotype of $A C R E$ overexpression plants challenged with Cladosporium fulvum infections should help confirm their role in the regulation of plant defense.

F-box-type E3 ligases have been implicated in the regulation of a variety of plant developmental processes [46]. Among the members of the F-box-type E3s implicated in plant defense signaling, the Coronatine Insensitivel (COI1) gene is the best example from which detailed functional characterization is available. COII encodes a $66-\mathrm{kDa}$ protein with amino-terminal F-box motif and a leucine-rich repeats (LRR) domain close to the carboxy-terminal end [47]. COI1 is required for plant response to jasmonic acids (JAs), which regulate root growth, pollen fertility, wound healing, and defense against insects and pathogens [48, 49]. The coil mutant fails to express the defense-related gene plant defensin 1.2 (PDF1.2) and is susceptible to insect herbivory and to pathogens $[50,51]$. Thus, it is believed that COI1 plays a positive role in the control of plant defense. Importantly, mutation of the tomato COII homolog LeCoil (also called jail) resulted in deficiency in multiple processes in tomato, including sensitivity to JAs, resistance to herbivores, glandular trichome development, and seed maturation [52], suggesting that COI1 function is largely conserved among different plant species. The COI1 protein associated with AtCUL1, AtRbx1, and either of the Arabidopsis Skp1-like proteins ASK1 or ASK2 to assemble ubiquitin-ligase complexes $\mathrm{SCF}^{\mathrm{COI} 1}$ in controlling JAs responses [ 53,54$]$. It is hypothesized that after activated by the JA signals, the $\mathrm{SCF}^{\mathrm{COII}} \mathrm{E} 3$ ligase targets regulators of JA-signaling pathways for ubiquitination, which leads to either modification of their activity or their degradation. Two putative candidate substrates of the $\mathrm{SCF}^{\mathrm{COII}} \mathrm{E} 3$ ligase have been identified recently and their functional characterization deepened our understanding of COI1-dependent plant defense signaling $[55,56]$. The histone deacetylase RPD3b (reduced potassium dependency $3 b$ ) was found to interact with COI1 in yeast two-hybrid screens, suggesting that it might be a COI1 target [55]. Histone deacetylation 
is involved in chromatin remodeling and is believed to reduce the transcription of the genes in the region where chromatin is modified [57]. Therefore, it is likely that $\mathrm{SCF}^{\mathrm{COI}}$-mediated modification or breakdown of RPD3b will derepress JA-dependent gene expression. In screening for suppressors of the coil mutant phenotype, Xiao et al. (2004) identified a mutation called cos 1 (coil suppressor 1 ) that can partly restore JA-related phenotype in the coil mutant [56]. COS1 encodes lumazine synthase, a component of the riboflavin pathway, and could be another putative target of $\mathrm{SCF}^{\mathrm{COI1}} \mathrm{E} 3$ ligase. SON1 is another F-box protein that has been implicated in plant defense signaling [58]. The son1 (suppressor of nim 1-1) mutant was isolated from genetic screens for suppressors of non-inducible immunity 1 (nim 1, also referred as nprl), of which the corresponding wild-type gene is a key positive regulator of Arabidopsis SAR $[59,60]$. The son1 mutant displays SAR-independent, constitutive resistance against both the virulent oomycete Peronospora parasitica and the bacterial pathogen Pseudomonas syringae pv tomato strain DC3000, indicating that SON1-mediated ubiquitination negatively regulate plant defense. The observation that suppression of SKP1 in tobacco plants by a gene-silencing strategy leads to the loss of $N$-mediated resistance to TMV further supports the notion that F-box proteins and their cognate SCF E3 ligases play a major role in plant defense signaling [61].

\section{U-box-type E3 ligases are newly identified members of the ubiquitination system involved in plant de- fense}

As discussed earlier, the U-box-containing proteins are newly identified E3 ubiquitin ligases that are present in yeast, plants, animals, and humans [35, 62, 63]. However, the number of U-box proteins varies among different organisms with a larger number of such proteins existing in the plant genomes. Less than 20 U-box proteins were predicted in the human genome [64]. Analyses of the rice fungal pathogen Magnaporthe grisea genome identified only two putative U-box proteins (L. Zeng, unpublished). In contrast, 59 and 78 U-box proteins were identified in the Arabidopsis (http://www.arabidopsis.org/info/genefamily/pub.html) and rice genomes (L Zeng, unpublished), respectively [65]. The uneven distribution of U-box proteins across different kingdoms prompts the speculation that the major role of plant U-box proteins is to signal responses to biotic and abiotic stresses [64]. However, the diversity of protein domains and motifs presented in rice and Arabidopsis Ubox proteins argued that these could also play critical roles other than stress control (L Zeng, unpublished). To date, several U-box proteins have been shown to be involved in plant defense against biotic and abiotic stresses (Table
1). The transcripts of U-box genes $C M P G 1$ and $C M P G 2$ were shown to accumulate extremely rapidly in parsley cell cultures after elicitor treatment, and the accumulation was localized around the pathogen infection sites in plants inoculated with Phythophthora sojae [66]. Arabidopsis homologs of CMPG1 and CMPG2 showed a similar expression pattern after challenged by elicitors [67]. In both cases, the authors identified a cis-acting element in the promoters of the genes that showed rapid responsiveness to pathogen but not wounding. The $C M P G$ genes encode proteins with an amino-terminal U-box domain and a caboxy-terminal half-rich in leucine residues. Although a rapid activation of these genes in pathogen-treated tissue inferred that they might play an important role in disease resistance, how these genes contribute to plant defenses remains elusive.

In addition to biotic stresses, U-box proteins have also been implicated in plant abiotic stress responses (Table 1). To date, one of the best-studied U-box proteins is CHIP (carboxyl terminus of Hsc70-interacting protein) in mammals. The CHIP protein has an amino-terminal tetratricopeptide repeat (TPR) domain and carboxy-terminal U-box domain. In mammals, the CHIP protein interacts with molecular chaperones such as HSP70 and HSP90 family members and removes substrate proteins of chaperones from the folding pathway to the ubiquitin-proteasome pathway for degradation [68]. CHIP was shown to possess an E3 ubiquitin ligase activity $[69,70]$ and has been implicated in ubiquitination and proteasomal targeting of a variety of proteins, such as transcription factors SMAD and E2A [71, 72] and nitric oxide synthase [73, 74]. CHIP was also found to be able to promote formation of Lys63-linked polyubiquitin chains in collaboration with Uev1a-Ubc13 in vitro [75]. In plants, the Arabidopsis CHIP gene AtCHIP is upregulated upon temperature stress [76]. However, overexpression of AtCHIP in Arabidopsis rendered plants more sensitive to both low- and high-temperature treatments, suggesting that CHIP's function in plants is complex and it warrants further detailed characterization.

The rice spl11 mutant displays a constitutive cell death phenotype in the absence of pathogen challenge and it belongs to a collection of mutants designated as "lesion mimics" based on their spontaneous lesion phenotypes similar to the HR or disease symptoms $[77,78]$. The cell death phenotype of spll1 was shown to be controlled by a single recessive gene [79]. The spl11 mutant conferred elevated non-race-specific resistance to both $M$. grisea and Xanthomonas oryzae pv. oryzae, the causal pathogens of rice blast and bacterial blight diseases, respectively [80]. The enhanced resistance of the spll1 mutant to the two pathogens correlates with the constitutive expression of several defense-related genes and lesion development on 
Table 2 Oxidiative stress- or cell death- and defense-related genes with over 4.5-fold upregulation compared with the wild-type plant IR68 at different leaf lesion development stages in spl11

\begin{tabular}{|c|c|c|c|c|}
\hline \multirow[t]{2}{*}{ Probe sets } & \multicolumn{3}{|c|}{ Fold upregulation } & \multirow[t]{2}{*}{ Protein or homolog } \\
\hline & YL & FL & $\mathrm{OL}$ & \\
\hline \multicolumn{5}{|c|}{ Oxidiative stress or cell death-related } \\
\hline os_ORF009142_at & 1.0 & 2.5 & 4.9 & L-ascorbate oxidase \\
\hline os001035_at & 1.3 & 5.1 & 6.7 & Alcohol dehydrogenase $\mathrm{V}$ \\
\hline os001069_at & 0.7 & 2.8 & 5.2 & Catalase isozyme A \\
\hline os002264.1_f_at & 1.4 & 5.1 & 8.8 & Alcohol dehydrogenase short chain \\
\hline os004902_at & 1.2 & 5.2 & 4.6 & Glutathione-s-transferase \\
\hline os007966.1_at & 1.4 & 5.1 & 5.2 & Flavanone 3-hydroxylase-like protein \\
\hline os010415.1_i_at & 1.1 & 27.4 & 82.2 & Putative alcohol dehydrogenase \\
\hline os013624.1_f_at & 1.4 & 6.0 & 10.0 & Short chain of alcohol dehydrogenase-like \\
\hline os010304_at & 1.0 & 7.2 & 5.7 & Potassium transporter \\
\hline \multicolumn{5}{|l|}{ Defense-related } \\
\hline os_ORF008199_at & 5.4 & 8.1 & 5.5 & Extensin precursor \\
\hline os_ORF011125_at & 2.6 & 5.7 & 2.5 & Endochitinase 1 precursor \\
\hline os_ORF015958_at & 5.2 & 26.4 & 7.2 & Beta-1, 3-glucanase \\
\hline os000314_r_at & 3.0 & 28.8 & 20.5 & Rice PR-5 \\
\hline os000349_at & 1.8 & 9.4 & 7.4 & PR-3 \\
\hline os000452_at & 1.2 & 9.3 & 9.5 & Soybean p21 (thamatin-like) \\
\hline os000536_s_at & 1.1 & 22.9 & 17.3 & PR-1-like \\
\hline os000590_f_at & 1.6 & 6.2 & 6.8 & Endochitinase precursor \\
\hline os000608.1_at & 1.3 & 13.9 & 13.1 & Hevein-like protein procursor \\
\hline os000959.1_at & 2.9 & 10.5 & 2.0 & Beta-1,3-glucanase (isoenzyme V) \\
\hline os001265_f_at & 1.2 & 11.5 & 7.9 & PR-1 precursor \\
\hline os001563.1_at & 2.3 & 21.2 & 30.1 & PBZ1 \\
\hline os002484_f_at & 4.5 & 7.2 & 5.3 & Extensin precursor-like \\
\hline os002683_at & 1.1 & 35.2 & 19.6 & Subtilisin-chymotrypsin inhibitor-2-likevvv \\
\hline os002953.1_at & 2.0 & 5.8 & 3.6 & Anthranilate synthase \\
\hline os003151_f_at & 1.0 & 5.1 & 6.8 & Class IV chitinase \\
\hline os003773_at & 1.7 & 6.9 & 4.2 & PR-3 (type Q) \\
\hline os008091_f_at & 1.1 & 1.8 & 9.1 & Basic endochitinase 2 precursor \\
\hline os011227.1_at & 1.3 & 8.3 & 2.6 & Dehydration-responsive protein RD22-like \\
\hline os012858_s_at & 2.0 & 5.3 & 3.6 & Anthranilate synthase component II \\
\hline os015959_at & 1.1 & 4.9 & 2.8 & Beta-glucosidase-like \\
\hline os016332_i_at & 1.0 & 6.9 & 11.0 & Subtilisin-like protease inhibitor \\
\hline os_ORF014847_at & 1.1 & 8.5 & 17.4 & Subtilisin-like protease inhibitor \\
\hline os016724_i_at & 1.5 & 4.1 & 4.6 & Zeamatin \\
\hline os017465_at & 1.1 & 5.7 & 8.4 & Beta-glucosidase \\
\hline os024957.1 at & 6.2 & 1.0 & 0.6 & Salt-stress-induced protein-like \\
\hline
\end{tabular}


the leaves [80]. The constitutive activation of defense responses in the spl11 mutant was further confirmed when an array-based expression profiling was performed using the Sygenta rice gene chips that harbor nearly half of all rice genes [81] to analyze the impact of the spl11 mutation on the rice transcriptome. Comparison of the global transcripts between the wild-type plants and the spl11 mutant plants in three types of leaf tissues with different lesion development phenotypes revealed that the spl11 mutation causes significant changes in the rice transcriptome (data not shown). Over 300 genes were highly induced in the fully expanded leaves of spl11. Nearly half of the highly induced genes are classified as oxidative stress/cell death- or defense-related genes (Table 2 and data not shown). The Spl11 gene was isolated by a map-based cloning strategy and it encodes a protein with a central U-box domain and carboxy-terminal armadillo (ARM) repeats domain [82, 83]. SPL11 homologs were found in several other plant species but not in human, animals, and yeast, suggesting that SPL11 might be unique to plants. In vitro ubiquitination assay indicated that the SPL11 protein possesses E3 ubiquitin ligase activity that is dependent on an intact U-box domain, suggesting a role of the ubiquitination system in the control of plant cell death and defense [83]. We speculate that SPL11 may target one or more regulators that promote $\mathrm{PCD}$ and defense signaling pathways. In attempting to identify such substrate proteins and other components potentially involved in Spl11-mediated cell death and defense signaling, we have isolated several SPL11-interacting proteins in yeast twohybrid screens (L. Zeng et al., unpublished). Two of the proteins that showed strong interaction with SPL11 were found to be homologous to pre-mRNA processing proteins, inferring that SPL11 might regulate plant cell death and defense through the control of alternative splicing of the transcripts of cell death and/or defense regulators. The ubiquitination machinery has been demonstrated to be involved in the control of general transcription [84]. But the involvement of ubiquitination in the control of processing specific transcripts has not yet been reported. Functional characterization of the SPL11 interactors should shed light on the molecular basis by which ubiquitination system contributes to plant defense signaling and regulation.

\section{Other components of the ubiquitination system are also involved in plant defense signaling}

Evidence on the involvement of other components in the ubiquitination system in plant defense signaling came from the genetic analysis of the SCF complexes in Arabidopsis. Several components that potentially regulate E3 ligase activity were implicated in plant defenses in these studies (Table 1). The RUB (related to ubiquitin, also known as
Nedd8) is a ubiquitin-like protein that covalently attaches to Cullins, the core subunit of the SCF E3 ligase complex. Rub1/Nedd8 and consequently its conjugation pathway are required for the SCF E3 activity $[85,86]$. Mutation of $R C E 1$ (RUB-conjugating enzyme1) resulted in defects in the expression of defense-related genes' basic chitinase and $P D F 1.2$, inferring that the SCF E3 complex it modified is required for plant defense [87]. RCE1 was shown in Arabidopsis to be part of a stable $\mathrm{SCF}^{\mathrm{TIR}}$ ubiquitin E3 ligase complex [88], which is a critical component in plant auxin signaling [89-91]. It will be intriguing to see if RCE1 is also associated with $\mathrm{SCF}^{\mathrm{COI} 1}$ complex, which has been shown be involved in plant defense signaling (see above).

The plant SGT1 (Suppressor of G2 allele of skp1) gene was first isolated independently by two laboratories; one in the searching for interacting proteins for the key signaling component of multiple $R$-gene-mediated resistance, RAR1[12], and the other in the screening for the genes essential for $R$ gene RPP5-mediated disease resistance, respectively $[92,93]$. Studies from several groups indicated that $S G T 1$ is required by a diverse range of $R$ genes from different plant species in response to a variety of pathogens [61, 92-95]. In yeast, SGT1 is a key regulatory protein in centromere function and cell cycle transition and is associated with SKP1 to regulate the activity of the SCF E3 ligase complex [96]. Thus it is speculated that SGT1 functions in plant defense by regulating the activity of the yet unknown SCF E3 ligase that regulates plant defense signal transduction. This is partly supported by the observation that the Arabidopsis SGT1 gene could complement yeast sgt1 mutations and that SGT1 is associated with SCF subunits SKP1 and CUL1 in barley and tobacco cell extracts $[61,93]$. The finding that $S G T 1$ is required for $\mathrm{SCF}^{\mathrm{TIR}}$-mediated plant auxin signaling indicated that it is involved in the regulation of plant physiological processes other than defense responses and also supported the notion that the mechanism by which SGT1 functions might be conserved across kingdoms [97]. Nevertheless, it is unlikely that $\mathrm{SCF}^{\mathrm{TIR}}$ is the E3 ligase related to SGT1 in the control of $R$-gene-mediated resistance. It is possible that several different F-box proteins and their cognate SCFs associate with SGT1 and that each controls the signal transduction mediated by different subset of $R$ genes. Identification of such F-box protein(s) will be instrumental to an in-depth understanding of the molecular mechanism underlying SGT1-regulated, R-gene-mediated disease resistance.

At present, it is unclear how SGT1 regulates the activity of SCF E3 ligase. However, several pieces of evidence might provide some clues to this question. Takahashi et al. (2003) demonstrated that barley SGT1 (HvSGT1) and Arabidopsis SGT1b (AtSGT1b) both interacted with barley cytosolic heat shock protein 90 (HvHSP90) in yeast cells 
and HvHSP90 co-immuno-precipitated with HvSGT1 in barley plants [98]. The HSP90 proteins are members of the heat shock protein superfamily that are implicated in protein complex maturation, in the activation of innate immune system, and in regulating the activity of many signal transduction proteins [99-101]. In animals, SGT1 was demonstrated to share structural features with HSP90 co-chaperones of the p23 families [102], which prompted the speculation that SGT1 might have a co-chaperone function in facilitating SCF complex assembly. In Arabidopsis, mutation of SGT1 did not affect the assembly of the $\mathrm{SCF}^{\mathrm{TIR}}$ complex [97]. This argues that SGT1, rather than facilitating the assembly of the SCF complex, might regulate the SCF activity by other means such as assisting in the ubiquitination process once the substrate is bound. Recent genetic analyses from the Dangl group suggested that Arabidopsis SGT1b has two genetically separable functions in some $R$-gene-mediated resistance pathways, being a positive regulator of $R$-gene-induced HR cell death and a negative regulator in pre-activated $\mathrm{R}$ protein accumulation, which is antagonized by both RAR1 and HSP90 [103]. They proposed that SGT1b might associate with SCF in targeting HSP90-associated R proteins for ubiquitinationmediated degradation.

The COP9 (constitutively photomorphogenic 9) signalosome (CSN), a conserved multi-protein complex involved in light-dependent plant morphogenesis and other plant developmental pathways, shows remarkable similarity to the lid subcomplex of the 19S RP $[104,105]$. It is speculated that CSN might substitute for the lid of the 19S RP in creating new forms of $26 \mathrm{~S}$ proteasome with distinct specificities [22]. Subunits of the COP9 signalosome are required for $\mathrm{N}$-gene-mediated resistance to TMV [61]. Multiple lines of evidence support the notion that COP9 is a regulator of SCF and other Cullin-based ubiquitin ligases by cleavage of the Nedd8 from the CUL1 of the SCF complex [86, 106, 107], suggesting that COP9 plays its role in plant defense through the ubiquitination system. The interaction of the COP9 signalosome with SGT1 and RAR1 in vivo further support this hypothesis [61, 93].

\section{Pathogen-derived proteins act as E3 ligases in host cells}

In the last few years, several studies have demonstrated that some viral proteins act as E3 ligases in host cells. The MIR proteins of Kaposi's sarcoma-associated virus (KSHV) regulate the accumulation and trafficking of target membrane proteins by acting as E3 ubiquitin ligases [108]. The same group recently reported that MIR1, but not MIR2, promoted downregulation of MHC I molecules lacking lysine residues in their intracytoplasmic domain. In the presence of MIR1, these MHC I molecules were ubiquitinated, and their association with ubiquitin was sensitive to $\beta$-mercaptoethanol, unlike lysine-ubiquitin bonds [109]. Similarly, the RING-finger proteins MBP-IAP2, IE2, and PE38 of Bombyx mori nucleopolyhedrovirus (BmNPV) are able to reconstitute ubiquitination activity in vitro and their activity is dependent on their RING-finger motif [110]. Interestingly, Huang et al. [111] demonstrated that the 28 proteins encoded by the ectromelia virus and the variola virus possess E3 ubiquitin ligase activity in biochemical assays as well as in cultured mammalian cells. Point mutations disrupting the RING-finger domain of $\mathrm{p} 28$ completely abolish its E3 ligase activity. In addition, p28 functions cooperatively with $\mathrm{Ubc} 4$ and $\mathrm{UbcH} 5 \mathrm{c}$, the E2-conjugating enzymes involved in $26 \mathrm{~S}$ proteasome degradation of protein targets. Whether any plant viral proteins could act as an E3 ligase during infection has yet to be reported.

During the infection process, pathogens deliver various virulence proteins into host cells to modulate diverse biochemical processes in plants [112]. In nature, infection of plants by Agrobacterium, a soil-borne plant pathogenic bacterium, causes crown-gall disease [113]. The transfer of Agrobacterium-transferred DNA (T-DNA) segment from the bacterial tumor-inducing (Ti) plasmid into the host cell, its integration into the host genome, and the expression of its encoded genes are the major steps of the Agrobacterium infection process [113]. The bacterial-transferred (T) DNA is believed to be imported into the host cell nucleus as a DNA-protein complex (T-complex), within which the associated bacterial virulence protein such as VirD2 and VirE2 mediate the nuclear import of the T-DNA [114]. Host proteins, such as VIP1, have also been demonstrated to be involved in the T-DNA-importing process [115]. The associated proteins must be uncoated from T-DNA before it is integrated into plant genome. Recent study showed that VirF, one of the few known exported Vir proteins from Agrobacterium is involved in targeted proteolysis of VIP1 and VirE2, probably facilitating the uncoating and integration process [116]. Experiments in yeast and in planta revealed that VIP1 and its cognate VirE2 are specifically targeted by the VirF-containing Skp1-Cdc53-cullin-F-box complex for proteolysis. Direct evidence on how the bacterial-secreted proteins act as an E3 ligase in plant cells and affect the host defense response came from a recent study by Janjusevic et al. [117]. When the Pseudomonas syringae-type III effector AvrPtoB is delivered into tomato plants containing the Pto resistance gene, it elicits PCD that inhibits pathogen invasion. In contrast, AvrPtoB suppresses programmed cell death (PCD) in the Pto-absent, $R s b$-containing plants, leading to rapid pathogen spread in the infected tissues. Structural analysis indicated that the C-terminal region of AvrPtoB is highly homologous to the 
U-box and RING-finger domain of eukaryotic E3 ligases. In vitro $\mathrm{E} 3$ ligase activity assay indicated that it has ubiquitin ligase activity. Mutation of the conserved residues in the U-box/RING-finger motif of AvrPtoB significantly compromised AvrPtoB's anti-PCD activity in tomato leaves and dramatically reduced disease symptom on infected plants. These results clearly demonstrated that $P$. syringae use an E3 ligase effector protein to suppress plant PCD and probably other defense-related processes in the infected cells. Identification of the associated E2 enzyme and potential host substrate of AvrPtoB involved in the interaction will be crucial in the future studies.

\section{Conclusions}

In the past few years, we have witnessed the identification of different components of the ubiquitin/26S proteasome system that are involved in plant-pathogen interactions. The emerging picture suggested that ubiquitination modification might occur at different levels of the defense responses in plants, from the $\mathrm{R}$ protein itself to downstream signaling components or regulators. In animals, the implication of the ubiquitination system in targeting key regulators in various processes has been well documented. For example, ubiquitination of key pro- and anti-apoptotic regulators was shown to be the principal mechanism underlying the regulation of apoptosis by the ubquitination/26S proteasome system [118]. In this sense, it will not be surprising that key plant defense signaling components or regulators will be identified as the substrates of the ubiquitination system in the near future. Moreover, the extreme importance of the ubiquitination system in regulating a wide range of cellular processes necessitates fine-tuned spatial and temporal occurrences of such modifications. Thus, understanding of when and where (i.e., at what level) ubiquitination operates in regulating plant defenses could be of equal importance to the determination of which substrates are targeted. It is noteworthy that ubiquitinated substrate proteins not necessarily are targeted for degradation [119]. Mono-ubiquitination or non-canonical ubiquitination might also be employed to fine tune the function of modified substrate proteins in plant-pathogen interactions. The recent exciting finding that plant pathogens deliver E3-like effectors into plant cell raises other intriguing questions such as how these E3 ligase proteins from pathogens interfere with the activation and signaling of host defense responses. Identification and functional characterization of their substrates in the host cells will definitely provide new insights into the molecular mechanisms associated with plant recognition of the pathogen and subsequent activation of defense responses.

\section{Acknowledgements}

The authors thank Hei Leung, Alicia Bordeos, Marietta Baraoidan and GS Khush at the International Rice Research Institute (IRRI) for providing the spl11 allelic mutants and other genetic materials, and Sherman Chang at Syngenta for performing the microarray hybridizations. Special thanks to Hong Ma for his editing of the manuscript and to the ad hoc reviewer for his/her helpful comments. This project was in part supported by the IRRI-USAID Linkage Program.

\section{References}

1 Thordal-Christensen H. Fresh insights into processes of nonhost resistance. Curr Opin Plant Biol 2003; 6:351-357.

2 Dangl JL, Jones DGJ. Plant pathogens and integrated defence responses to infection. Nature $2001 ; \mathbf{4 1 1}: 826-833$.

3 Nürnberger T, Brunner F, Kemmerling B, Piater L. Innate immunity in plants and animals: striking similarities and obvious differences. Immunol Rev 2004; 198:249-266.

4 Bonas U, Lahaye T. Plant disease resistance triggered by pathogen-derived molecules: refined models of specific recognition. Curr Opin Microbiol 2002; 5:44-50.

5 Flor HH. Current status of the gene-for-gene concept. Annu Rev Phytopathol 1971; 9:275-296.

6 Heath MC. Hypersensitive response-related death. Plant Mol Biol 2000; 44:321-334.

7 Durrant WE, Dong X. Systemic acquired resistance. Annu Rev Phytopathol 2004; 42:185-209.

8 Van der Hoorn RA, De Wit PJ, Joosten MH. Balancing selection favors guarding resistance proteins. Trends Plant Sci 2002; 7:67-71.

9 Mackey D, Belkhadir Y, Alonso JM, Ecker JR, Dangl JL. Arabidopsis RIN4 is a target of the type III virulence effector AvrRpt2 and modulates RPS2-mediated resistance. Cell 2003; 112:379-389.

10 Schulze-Lefert P, Bieri S. Plant sciences. Recognition at a distance. Science 2005; 308:506-508.

11 Parker JE, Holub EB, Frost LN, et al. Characterization of eds1, a mutation in Arabidopsis suppressing resistance to Peronospora parasitica specified by several different RPP genes. Plant Cell 1996; 8:2033-2046.

12 Shirasu K, Lahaye T, Tan MW, et al. A novel class of eukaryotic zinc-binding proteins is required for disease resistance signaling in barley and development in C. elegans. Cell 1999; 99:355366.

13 Nimchuk Z, Eulgem T, Holt BF, 3rd, Dangl JL. Recognition and response in the plant immune system. Annu Rev Genet 2003; 37:579-609.

14 Marx J. Ubiquitin lives up to its name. Science 2002; 297:17921794.

15 Smalle J, Vierstra RD. The ubiquitin 26s proteasome proteolytic pathway. Annu Rev Plant Biol 2004; 55:555-590.

16 Voges D, Zwickl P, Baumeister W. The 26S proteasome: a molecular machine designed for controlled proteolysis. Annu Rev Biochem 1999; 68:1015-1068. 
17 Glickman MH, Rubin DM, Fried VA, Finley D. The regulatory particle of the Saccharomyces cerevisiae proteasome. Mol Cell Biol 1998; 18:3149-3162.

18 Ferrell K, Wilkinson CR, Dubiel W, Gordon C. Regulatory subunit interactions of the $26 \mathrm{~S}$ proteasome, a complex problem. Trends Biochem Sci 2000; 25:83-88.

19 Glickman MH, Raveh D. Proteasome plasticity. FEBS Lett 2005; 579:3214-3223.

20 Hartmann-Petersen R, Seeger M, Gordon C. Transferring substrates to the $26 \mathrm{~S}$ proteasome. Trends Biochem Sci 2003; 28:26-31.

21 Kraft E, Stone SL, Ma L, et al. Genome Analysis and Functional Characterization of the E2 and RING-Type E3 Ligase Ubiquitination Enzymes of Arabidopsis. Plant Physiol 2005; 139:1597-1611.

22 Vierstra RD. The ubiquitin/26S proteasome pathway, the complex last chapter in the life of many plant proteins. Trends Plant Sci 2003; 8:135-142.

23 Ciechanover A. The ubiquitin-proteasome pathway: on protein death and cell life. EMBO J 1998; 17:7151-7160.

24 Huibregtse JM, Scheffner M, Beaudenon S, Howley PM. A family of proteins structurally and functionally related to the E6-AP ubiquitin-protein ligase. Proc Natl Acad Sci USA 1995; 92:2563-2567.

25 Scheffner M, Huibregtse JM, Vierstra RD, Howley PM. The HPV-16 E6 and E6-AP complex functions as a ubiquitin-protein ligase in the ubiquitination of p53. Cell 1993; 75:495-505.

26 Zheng N, Schulman BA, Song L, et al. Structure of the Cul1Rbx1-Skp1-F boxSkp2 SCF ubiquitin ligase complex. Nature 2002; 416:703-709.

27 Stebbins CE, Kaelin WG, Jr., Pavletich NP. Structure of the VHL-ElonginC-ElonginB complex: implications for VHL tumor suppressor function. Science 1999; 284:455-461.

28 Kurz T, Pintard L, Willis JH, et al. Cytoskeletal regulation by the Nedd8 ubiquitin-like protein modification pathway. Science 2002; 295:1294-1298.

29 Pintard L, Willems A, Peter M. Cullin-based ubiquitin ligases: Cul3-BTB complexes join the family. EMBO J 2004; 23:16811687.

30 Gagne JM, Downes BP, Shiu S-H, Durski AM, Vierstra RD. The F-box subunit of the SCF E3 complex is encoded by a diverse superfamily of genes in Arabidopsis. Proc Natl Acad Sci USA 2002; 99:11519-11524.

31 Shen WH, Parmentier Y, Hellmann H, et al. Null mutation of AtCUL1 causes arrest in early embryogenesis in Arabidopsis. Mol Biol Cell 2002; 13:1916-1928.

32 Downes BP, Stupar RM, Gingerich DJ, Vierstra RD. The HECT ubiquitin-protein ligase (UPL) family in Arabidopsis: UPL3 has a specific role in trichome development. Plant J 2003; 35:729742.

33 Stone SL, Hauksdóttir H, Troy A, et al. Functional analysis of the RING-type ubiquitin ligase family of Arabidopsis. Plant Physiol 2005; 137:13-30.

34 Joazeiro CA, Weissman AM. RING finger proteins: mediators of ubiquitin ligase activity. Cell 2000; 102:549-552.

35 Koegl M, Hoppe T, Schlenker S, et al. A novel ubiquitination factor, E4, is involved in multiubiquitin chain assembly. Cell 1999; 96:635-644.

36 Aravind L, Koonin EV. The U box is a modified RING finger
- a common domain in ubiquitination. Curr Biol 2000; 10: R132-R134.

37 Ohi MD, Vander Kooi CW, Rosenberg JA, Chazin WJ, Gould KL. Structural insights into the U-box, a domain associated with multi-ubiquitination. Nat Struct Biol 2003; 10:250-255.

38 Boyes DC, Nam J, Dangl JL. The Arabidopsis thaliana RPM1 disease resistance gene product is a peripheral plasma membrane protein that is degraded coincident with the hypersensitive response. Proc Natl Acad Sci USA 1998; 95:15849-15854.

39 Salinas-Mondragon RE, Garciduenas-Pina C, Guzman P. Early elicitor induction in members of a novel multigene family coding for highly related RING-H2 proteins in Arabidopsis thaliana. Plant Mol Biol 1999; 40:579-590.

40 Serrano M, Guzman P. Isolation and gene expression analysis of Arabidopsis thaliana mutants with constitutive expression of ATL2, an early elicitor-response RING-H2 zinc-finger gene. Genetics 2004; 167:919-929.

41 Molnar G, Bancos S, Nagy F, Szekeres M. Characterisation of BRH1, a brassinosteroid-responsive RING-H2 gene from Arabidopsis thaliana. Planta 2002; 215:127-133.

42 Takai R, Matsuda N, Nakano A, et al. EL5, a rice N-acetylchitooligosaccharide elicitor-responsive RING-H2 finger protein, is a ubiquitin ligase which functions in vitro in co-operation with an elicitor-responsive ubiquitin-conjugating enzyme, OsUBC5b. Plant J 2002; 30:447-455.

43 Takizawa M, Goto A, Watanabe Y. The tobacco ubiquitin-activating enzymes NtE1A and NtE1B are induced by tobacco mosaic virus, wounding and stress hormones. Mol Cells 2005; 19:228-231.

44 Ramonell K, Berrocal-Lobo M, Koh S, et al. Loss-of-function mutations in chitin responsive genes show increased susceptibility to the powdery mildew pathogen Erysiphe cichoracearum. Plant Physiol 2005; 138:1027-1036.

45 Rowland O, Ludwig AA, Merrick CJ, et al. Functional analysis of Avr9/Cf-9 rapidly elicited genes identifies a protein kinase, $\mathrm{ACIK} 1$, that is essential for full Cf-9-dependent disease resistance in tomato. Plant Cell 2005; 17:295-310.

46 Sullivan JA, Shirasu K, Deng XW. The diverse roles of ubiquitin and the $26 \mathrm{~S}$ proteasome in the life of plants. Nat Rev Genet 2003; 4:948-958.

47 Xie DX, Feys BF, James S, Nieto-Rostro M, Turner JG. COI1: an Arabidopsis gene required for jasmonate-regulated defense and fertility. Science 1998; 280:1091-1094.

48 Devoto A, Ellis C, Magusin A, et al. Expression profiling reveals COI1 to be a key regulator of genes involved in wound- and methyl jasmonate-induced secondary metabolism, defence, and hormone interactions. Plant Mol Biol 2005; 58:497-513.

49 Turner JG, Ellis C, Devoto A. The jasmonate signal pathway. Plant Cell 2002; 14 Suppl:S153-S164.

50 McConn M, Creelman RA, Bell E, Mullet JE, Browse J. Jasmonate is essential for insect defense in Arabidopsis. Proc Natl Acad Sci USA 1997; 94:5473-5477.

51 Thomma B, Eggermont K, Penninckx I, et al. Separate jasmonate-dependent and salicylate-dependent defense-response pathways in Arabidopsis are essential for resistance to distinct microbial pathogens. Proc Natl Acad Sci USA 1998; 95:1510715111.

52 Li L, Zhao Y, McCaig BC, et al. The tomato homolog of CORONATINE-INSENSITIVE1 is required for the maternal control 
of seed maturation, jasmonate-signaled defense responses, and glandular trichome development. Plant Cell 2004; 16:126143.

53 Ren C, Pan J, Peng W, et al. Point mutations in Arabidopsis Cullin1 reveal its essential role in jasmonate response. Plant J 2005; 42:514-524

$54 \mathrm{Xu} \mathrm{L}$, Liu F, Lechner E, et al. The $\mathrm{SCF}^{\mathrm{CO} 11}$ ubiquitin-ligase complexes are required for jasmonate response in Arabidopsis. Plant Cell 2002; 14:1919-1935.

55 Devoto A, Nieto-Rostro M, Xie D, et al. COI1 links jasmonate signalling and fertility to the SCF ubiquitin-ligase complex in Arabidopsis. Plant J 2002; 32:457-466.

56 Xiao S, Dai L, Liu F, et al. COS1: an Arabidopsis coronatine insensitivel suppressor essential for regulation of jasmonatemediated plant defense and senescence. Plant Cell 2004; 16:1132-1142.

57 Carrozza MJ, Utley RT, Workman JL, Cote J. The diverse functions of histone acetyltransferase complexes. Trends Genet 2003; 19:321-329.

58 Kim HS, Delaney TP. Arabidopsis SON1 is an F-box protein that regulates a novel induced defense response independent of both salicylic acid and systemic acquired resistance. Plant Cell 2002; 14:1469-1482.

59 Ryals J, Weymann K, Lawton K, et al. The Arabidopsis NIM1 protein shows homology to the mammalian transcription factor inhibitor I kappa B. Plant Cell 1997; 9:425-439.

60 Cao H, Glazebrook J, Clarke JD, Volko S, Dong X. The Arabidopsis NPR1 gene that controls systemic acquired resistance encodes a novel protein containing ankyrin repeats. Cell 1997; 88:57-63.

61 Liu Y, Schiff M, Serino G, Deng XW, Dinesh-Kumar SP. Role of SCF ubiquitin-ligase and the COP9 signalosome in the $N$ gene-mediated resistance response to Tobacco mosaic virus. Plant Cell 2002; 14:1483-1496.

62 Meacham GC, Patterson C, Zhang W, Younger JM, Cyr DM. The Hsc70 co-chaperone CHIP targets immature CFTR for proteasomal degradation. Nat Cell Biol 2001; 3:100-105.

63 Stone SL, Anderson EM, Mullen RT, Goring DR. ARC1 is an E3 ubiquitin ligase and promotes the ubiquitination of proteins during the rejection of self-incompatible Brassica pollen. Plant Cell 2003; 15:885-898.

64 Patterson C. A new gun in town: the $\mathrm{U}$ box is a ubiquitin ligase domain. Sci STKE 2002:PE4.

65 Azevedo C, Santos-Rosa MJ, Shirasu K. The U-box protein family in plants. Trends Plant Sci 2001; 6:354-358.

66 Kirsch C, Logemann E, Lippok B, Schmelzer E, Hahlbrock K. A highly specific pathogen-responsive promoter element from the immediate-early activated CMPG1 gene in Petroselinum crispum. Plant J 2001; 26:217-227.

67 Heise A, Lippok B, Kirsch C, Hahlbrock K. Two immediate-early pathogen-responsive members of the AtCMPG gene family in Arabidopsis thaliana and the W-box-containing elicitor-response element of AtCMPG1. Proc Natl Acad Sci USA 2002; 99:9049-9054.

68 Ballinger CA, Connell P, Wu Y, et al. Identification of CHIP, a novel tetratricopeptide repeat-containing protein that interacts with heat shock proteins and negatively regulates chaperone functions. Mol Cell Biol 1999; 19:4535-4545.

69 Murata S, Minami Y, Minami M, Chiba T, Tanaka K. CHIP is a chaperone-dependent E3 ligase that ubiquitylates unfolded protein. EMBO Rep 2001; 2:1133-1138.

70 Jiang J, Ballinger CA, Wu Y, et al. CHIP is a U-box-dependent E3 ubiquitin ligase: identification of $\mathrm{Hsc} 70$ as a target for ubiquitylation. J Biol Chem 2001; 276:42938-43944.

71 Li L, Xin H, Xu X, et al. CHIP mediates degradation of Smad proteins and potentially regulates Smad-induced transcription. Mol Cell Biol 2004; 24:856-864.

72 Huang Z, Nie L, Xu M, Sun X-H. Notch-induced E2A degradation requires $\mathrm{CHIP}$ and $\mathrm{Hsc} 70$ as novel facilitators of ubiquitination. Mol Cell Biol 2004; 24:8951-8962.

73 Jiang J, Cyr D, Babbitt RW, Sessa WC, Patterson C. Chaperone-dependent regulation of endothelial nitric-oxide synthase intracellular trafficking by the co-chaperone/ubiquitin ligase CHIP. J Biol Chem 2003; 278:49332-49341.

74 Peng HM, Morishima Y, Jenkins GJ, et al. Ubiquitylation of neuronal nitric-oxide synthase by CHIP, a chaperone-dependent E3 ligase. J Biol Chem 2004; 279:52970-52977.

75 Zhang M, Windheim M, Roe SM, et al. Chaperoned ubiquitylation - crystal structures of the CHIP U box E3 ubiquitin ligase and a CHIP-Ubc13-Uev1a complex. Mol Cell 2005; 20:525-538.

76 Yan J, Wang J, Li Q, et al. AtCHIP, a U-box-containing E3 ubiquitin ligase, plays a critical role in temperature stress tolerance in Arabidopsis. Plant Physiol 2003; 132:861-869.

77 Hoisington DA, Neuffer MG, Walbot V. Disease lesion mimics in maize. I. Effect of genetic background, temperature, developmental age, and wounding on necrotic spot formation with Les 1. Dev Biol 1982; 93:381-388.

78 Dangl JL, Dietrich RA, Richberg MH. Death don't have no mercy: cell death programs in plant-microbe interactions. Plant Cell 1996; 8:1793-1807.

79 Singh K, Multani DS, Khush GS. A new spotted leaf mutant in rice. Rice Genet Newslet 1995; 12:192-193.

80 Yin Z, Chen J, Zeng L, et al. Characterizing rice lesion mimic mutants and identifying a mutant with broad-spectrum resistance to rice blast and bacterial blight. Mol Plant Microbe Interact 2000; 13:869-876.

81 Zhu T, Budworth P, Chen W, et al. Transcriptional control of nutrient partitioning during rice grain filling. Plant Biotech $\mathrm{J}$ 2003; 1:59-70.

82 Zeng L, Yin Z, Chen J, Leung H, Wang GL. Fine genetic mapping and physical delimitation of the lesion mimic gene Spl11 to a $160-\mathrm{kb}$ DNA segment of the rice genome. Mol Genet Genomics 2002; 268:253-261.

83 Zeng LR, Qu S, Bordeos A, et al. Spotted leaf11, a negative regulator of plant cell death and defense, encodes a U-box/armadillo repeat protein endowed with E3 ubiquitin ligase activity. Plant Cell 2004; 16:2795-2808.

84 Muratani M, Tansey WP. How the ubiquitin-proteasome system controls transcription. Nat Rev Mol Cell Biol 2003; 4:192201.

85 Gray WM, Hellmann H, Dharmasiri S, Estelle M. Role of the Arabidopsis RING-H2 protein RBX1 in RUB modification and SCF function. Plant Cell 2002; 14:2137-2144.

86 Lyapina S, Cope G, Shevchenko A, et al. Promotion of NEDDCUL1 conjugate cleavage by COP9 signalosome. Science 2001; 292:1382-1385.

87 Larsen PB, Cancel JD. A recessive mutation in the RUB1- 
conjugating enzyme, RCE1, reveals a requirement for RUB modification for control of ethylene biosynthesis and proper induction of basic chitinase and PDF1.2 in Arabidopsis. Plant J 2004; 38:626-638.

88 Dharmasiri S, Dharmasiri N, Hellmann H, Estelle M. The RUB/ Nedd8 conjugation pathway is required for early development in Arabidopsis. EMBO J 2003; 22:1762-1770.

89 Kepinski S, Leyser O. The Arabidopsis F-box protein TIR1 is an auxin receptor. Nature 2005; 435:446-451.

90 Dharmasiri N, Dharmasiri S, Estelle M. The F-box protein TIR1 is an auxin receptor. Nature $2005 ; \mathbf{4 3 5}: 441-445$.

91 Ruegger M, Dewey E, Gray WM, et al. The TIR1 protein of Arabidopsis functions in auxin response and is related to human SKP2 and yeast grr1p. Genes Dev 1998; 12:198-207.

92 Austin MJ, Muskett P, Kahn K, et al. Regulatory role of SGT1 in early R gene-mediated plant defenses. Science 2002; 295:20772080.

93 Azevedo C, Sadanandom A, Kitagawa K, et al. The RAR1 interactor SGT1, an essential component of R gene-triggered disease resistance. Science 2002; 295:2073-2076.

94 Peart JR, Lu R, Sadanandom A, et al. Ubiquitin ligase-associated protein SGT1 is required for host and nonhost disease resistance in plants. Proc Natl Acad Sci USA 2002; 99:10865-10869.

95 Tor M, Gordon P, Cuzick A, et al. Arabidopsis SGT1b is required for defense signaling conferred by several downy mildew resistance genes. Plant Cell 2002; 14:993-1003.

96 Kitagawa K, Skowyra D, Elledge SJ, Harper JW, Hieter P. SGT1 encodes an essential component of the yeast kinetochore assembly pathway and a novel subunit of the SCF ubiquitin ligase complex. Mol Cell 1999; 4:21-33.

97 Gray WM, Muskett PR, Chuang HW, Parker JE. Arabidopsis $\mathrm{SGT} 1 \mathrm{~b}$ is required for $\mathrm{SCF}^{\mathrm{TIR} 1}$-mediated auxin response. Plant Cell 2003; 15:1310-1319.

98 Takahashi A, Casais C, Ichimura K, Shirasu K. HSP90 interacts with RAR1 and SGT1 and is essential for RPS2-mediated disease resistance in Arabidopsis. Proc Natl Acad Sci USA 2003; 100:11777-11782.

99 Young JC, Agashe VR, Siegers K, Hartl FU. Pathways of chaperone-mediated protein folding in the cytosol. Nat Rev Mol Cell Biol 2004; 5:781-791.

100 Tsan MF, Gao B. Heat shock protein and innate immunity. Cell Mol Immunol 2004; 1:274-279.

101 Pratt WB, Toft DO. Regulation of signaling protein function and trafficking by the hsp90/hsp70-based chaperone machinery. Exp Biol Med (Maywood) 2003; 228:111-133.

102 Garcia-Ranea JA, Mirey G, Camonis J, Valencia A. p23 and HSP20/alpha-crystallin proteins define a conserved sequence domain present in other eukaryotic protein families. FEBS Lett 2002; 529:162-167.

103 Holt BF, 3rd, Belkhadir Y, Dangl JL. Antagonistic control of disease resistance protein stability in the plant immune system. Science 2005; 309:929-932.

104 Serino G, Deng XW. The COP9 signalosome: regulating plant development through the control of proteolysis. Annu Rev Plant Biol 2003; 54:165-182.

105 Wei N, Tsuge T, Serino G, et al. The COP9 complex is conserved between plants and mammals and is related to the $26 \mathrm{~S}$ proteasome regulatory complex. Curr Biol 1998; 8:919-922.

106 Cope GA, Suh GS, Aravind L, et al. Role of predicted metal- loprotease motif of Jab1/Csn5 in cleavage of Nedd8 from Cul1. Science 2002; 298:608-611.

107 Zhou C, Wee S, Rhee E, et al. Fission yeast COP9/signalosome suppresses cullin activity through recruitment of the deubiquitylating enzyme Ubp12p. Mol Cell 2003; 11:927-938.

108 Coscoy L, Sanchez DJ, Ganem D. A novel class of herpesvirus-encoded membrane-bound E3 ubiquitin ligases regulates endocytosis of proteins involved in immune recognition. J Cell Biol 2001; 155:1265-1273.

109 Cadwell K, Coscoy L. Ubiquitination on nonlysine residues by a viral E3 ubiquitin ligase. Science 2005; 309:127-130.

110 Imai N, Matsuda N, Tanaka K, et al. Ubiquitin ligase activities of Bombyx mori nucleopolyhedrovirus RING finger proteins. J Virol 2003; 77:923-930.

111 Huang J, Huang Q, Zhou X, et al. The poxvirus p28 virulence factor is an E3 ubiquitin ligase. J Biol Chem 2004; 279:5411054116.

112 Galan JE, Collmer A. Type III secretion machines: bacterial devices for protein delivery into host cells. Science 1999; 284:1322-1328.

113 Gelvin SB. Agrobacterium and plant genes involved in T-DNA transfer and integration. Annu Rev Plant Physiol Plant Mol Biol 2000; 51:223-256.

114 Ziemienowicz A, Merkle T, Schoumacher F, Hohn B, Rossi L. Import of Agrobacterium T-DNA into plant nuclei: two distinct functions of VirD2 and VirE2 proteins. Plant Cell 2001; 13:369383

115 Tzfira T, Vaidya M, Citovsky V. VIP1, an Arabidopsis protein that interacts with Agrobacterium VirE2, is involved in VirE2 nuclear import and Agrobacterium infectivity. EMBO J 2001; 20:3596-3607.

116 Tzfira T, Vaidya M, Citovsky V. Involvement of targeted proteolysis in plant genetic transformation by Agrobacterium. Nature 2004; 431:87-92.

117 Janjusevic R, Abramovitch RB, Martin GB, Stebbins CE. A bacterial inhibitor of host programmed cell death defenses is an E3 ubiquitin ligase. Science 2006; 311:222-226.

118 Lee JC, Peter ME. Regulation of apoptosis by ubiquitination. Immunol Rev 2003; 193:39-47.

119 Schnell JD, Hicke L. Non-traditional functions of ubiquitin and ubiquitin-binding proteins. J Biol Chem 2003; 278:3585735860 .

120 Durrant WE, Rowland O, Piedras P, Hammond-Kosack KE, Jones JDG. cDNA-AFLP reveals a striking overlap in racespecific resistance and wound response gene expression profiles. Plant Cell 2000; 12:963-977.

121 Vijayan P, Shockey J, Levesque CA, Cook RJ, Browse J. A role for jasmonate in pathogen defense of arabidopsis. Proc Natl Acad Sci U S A 1998; 95:7209-7214.

122 Banzai T, Hershkovits G, Katcoff DJ, et al. Identification and characterization of mRNA transcripts differentially expressed in response to high salinity by means of differential display in the mangrove, Bruguiera gymnorrhiza. Plant Sci 2002; 162:499505 .

123 Gidekel M, Destefano-Beltran L, Garcia P, et al. Identification and characterization of three novel cold acclimation-responsive genes from the extremophile hair grass Deschampsia antarctica Desv. Extremophiles 2003; 7:459-469.

124 Lee H, Xiong L, Gong Z, et al. The Arabidopsis HOS1 gene 
negatively regulates cold signal transduction and encodes a RING finger protein that displays cold-regulated nucleo-cytoplasmic partitioning. Genes Dev 2001; 15:912-924.

125 Feussner K, Feussner I, Leopold I, Wasternack C. Isolation of a cDNA coding for an ubiquitin-conjugating enzyme UBC1 of tomato - the first stress-induced UBC of higher plants. FEBS Lett 1997; 409:211-215.
126 Woo HR, Kim JH, Nam HG, Lim PO. The delayed leaf senescence mutants of Arabidopsis, ore1, ore3, and ore9 are tolerant to oxidative stress. Plant Cell Physiol 2004; 45:923-932.

127 Tang D, Christiansen KM, Innes RW. Regulation of plant disease resistance, stress responses, cell death, and ethylene signaling in Arabidopsis by the EDR1 protein kinase. Plant Physiol 2005; 138:1018-1026.

Edited by Hong Ma 